\title{
Mathematical Model of Environmental Pollution by Motorcar in an Urban Area
}

\author{
Valeriy Perminov \\ Belovo Branch of Kemerovo State University, \\ 652600 Belovo, Kemerovo region, Russia \\ pva@belovo.kemsu.ru
}

\begin{abstract}
In the present paper it is developed mathematical model for description of heat and mass transfer processes and predicting velocity, temperature and pollution concentrations near roadway. To describe convective transfer controlled by the wind and gravity, we use Reynolds equations for turbulent flow. The boundary value problem was solved numerically. A discrete analog for equations was obtained by means of the control volume method. Methods of predicting concentrations of automobile exhaust gases near roadways are needed for the planning and design of roads and nearby structures.
\end{abstract}

\section{Introduction}

Mathematical model for description of heat and mass transfer processes and predicting velocity, temperature and pollution concentrations near roadway is constructed as a result of an analysis of known experimental data and using concept and methods from reactive media mechanics [1] and existing environmental pollution models $[2,3]$. It is considered that 1) the flow has a developed turbulent nature, molecular transfer being neglected, 2) gaseous phase density doesn't depend on the pressure because of the low velocities of the flow in comparison with the velocity of the sound, 3) the traffic is uniformly distributed over all lanes, 4) two dimensional model used to predict the concentrations along a line normal to highway. The forest in forest belt represents a non-deformable porous-dispersed medium [4].

\section{Problem Formulation}

Let the coordinate reference point $x_{1}, x_{2}=0$ be situated at the center of the road surface source at the height of the roughness level, axis $O x_{2}$ directed upward, axis $O x_{1}$ directed parallel to the ground's surface to the right in the direction (Fig. 1).

The problem formulated above is reduced to a solution of the Reynolds and transport equations for turbulent flow:

$$
\frac{\partial \rho}{\partial t}+\frac{\partial}{\partial x_{j}}\left(\rho v_{j}\right)=0, j=1,2, i=1,2
$$




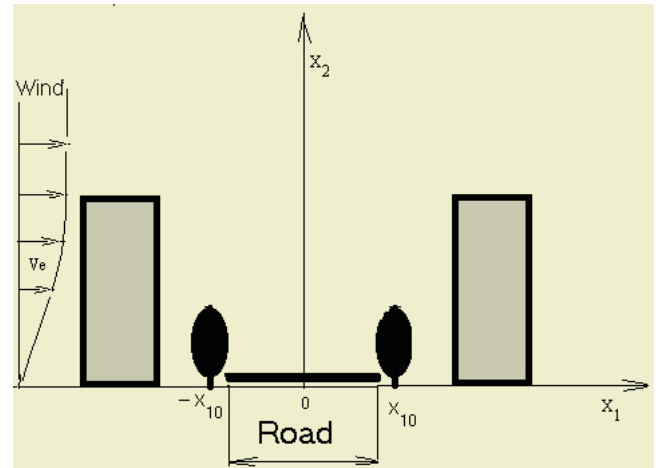

Fig. 1. Street canyon

$$
\begin{gathered}
\rho \frac{d v_{i}}{d t}=-\frac{\partial P}{\partial x_{i}}+\frac{\partial}{\partial x_{j}}\left(-\rho \vec{v}_{i}^{\prime} \vec{v}_{j}^{\prime}\right)-\rho s c_{d} v_{i}|\vec{v}|-\rho g_{i} \\
\rho c_{p} \frac{d T}{d t}=\frac{\partial}{\partial x_{j}}\left(-\rho c_{p} \overline{\left.v_{j}^{\prime} T^{\prime}\right)}-v_{2}\left(\rho_{e} g+c_{p} \rho \frac{d T_{e}}{d x_{2}}\right) ;\right. \\
\rho \frac{d c_{\alpha}}{d t}=\frac{\partial}{\partial x_{j}}\left(-\rho \overline{v_{j}^{\prime} c_{\alpha}^{\prime}}\right), \alpha=1,4 ; \\
\sum_{\alpha=1}^{5} c_{\alpha}=1, P_{e}=\rho R T \sum_{\alpha=1}^{5} \frac{c_{\alpha}}{M_{\alpha}}, \frac{\partial T_{e}}{\partial x_{2}}=\gamma_{e}, \vec{v}=\left(v_{1}, v_{2}\right), \vec{g}=(0, g) .
\end{gathered}
$$

The system of equations (1)-(5) must be solved taking into account the following initial and boundary conditions:

$$
\begin{gathered}
t=0: v_{1}=0, v_{2}=0, T=T_{e}, c_{\alpha}=c_{\alpha e}, T_{s}=T_{e} ; \\
x_{1}=-x_{1 e}: v_{1}=V_{e}\left(x_{2}\right), v_{2}=0, T=T_{e}, c_{\alpha}=c_{\alpha e} ; \\
x_{1}=x_{1 e}: \frac{\partial v_{1}}{\partial x_{1}}=0, \frac{\partial v_{2}}{\partial x_{1}}=0, \frac{\partial c_{\alpha}}{\partial x_{1}}=0, \frac{\partial T}{\partial x_{1}}=0 ; \\
x_{2}=0: v_{1}=0, v_{2}=V_{20}, T=T_{0}, c_{\alpha}=c_{\alpha 0},\left|x_{1}\right| \leq x_{10}, \\
v_{1}=0, v_{2}=0, T=T_{e}, c_{\alpha}=c_{\alpha e},\left|x_{1}\right|>x_{10} ; \\
x_{2}=x_{2 e}: \frac{\partial v_{1}}{\partial x_{2}}=0, \frac{\partial v_{2}}{\partial x_{2}}=0, \frac{\partial c_{\alpha}}{\partial x_{2}}=0, \frac{\partial T}{\partial x_{2}}=0 .
\end{gathered}
$$


Here and above $\frac{\mathrm{d}}{\mathrm{d} t}$ is the symbol of the total (substantial) derivative; $t$ is time; $x_{i}$, $v_{i},(i=1,2)$ are the Cartesian coordinates and the velocity components; $\rho, T$ - density and temperature of air, $P$ - pressure; $c_{P}$ - constant pressure specific heat of the gas, $c_{\alpha^{-}}$ mass concentrations $\left(\alpha=1-C O, 2-C_{x}, 3-N O_{x}, 4\right.$ - soot, 5 - inert components of air); $V_{e}$ - wind speed at the height $1,5 \mathrm{~m}, M_{\alpha}$ - molecular mass of $\alpha$-components of the gas phase; $V_{20}$ - velocity of automobile exhaust gases, $\gamma_{e}-$ gradient of stratification of temperature, $c_{d}$ is an empirical coefficient of the resistance of the vegetation, $s$ is the specific surface of the forest elements; index $e$ corresponds to the unperturbed parameters of the medium. The components of the tensor of turbulent stresses and the turbulent fluxes of heat and mass are written in terms of the gradients of the average flow [4]. The road is modeled as a plane source of burning motorway fuels with known temperature and concentrations of automobile exhaust gases.

\section{Numerical Simulation and Results}

The boundary value problem (1) - (10) was solved numerically. A discrete analog for equations was obtained by means of the control volume method using the SIMPLE algorithm [5]. The accuracy of the program was checked by the method of inserted analytical solutions. Analytical expressions for the unknown functions were substituted in (1)-(10) and the closure of the equations were calculated. Next, the values of the functions were inferred with an accuracy of not less than 1\%. Our practical situation requires calculation domain with obstacles in the middle of the flow region. It is convenient to employ a Cartesian coordinates but to modify the difference equations using method of fiction domain [5]. In the present calculations the results are obtained by using data: $T=300 \mathrm{~K}, V_{e}=2 \mathrm{~m} / \mathrm{sec}$, the width of highway is $12 \mathrm{~m}$, the number of cars passing per unit time (hour) is 3000, percentage structure of traffic (cars - 56\%, lorries and buses $-19 \%$, automobiles with diesel engine $-25 \%$ ). The distribution of

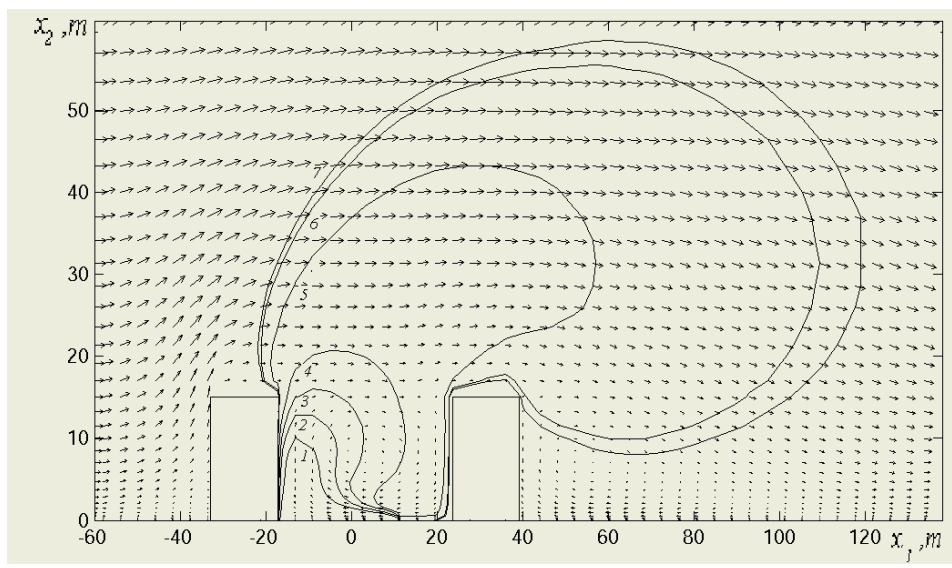

Fig. 2. Distributions of the vector velocity field and the concentration of carbon monoxide; 1 $1-5,0,2-2,0,3-1,0,4-0,5,5-0,1,6-0,05,7-0.04 \mathrm{mg} / \mathrm{m}^{3}$ 
temperature of air, velocity, concentrations of components were obtained at different instants of time. The fields of mass concentrations of automobile emissions $C O$ and vectorial fields of velocity (Fig. 2) were presented at the moment $t=6 \mathrm{~min}$ when the steady situation is realized. The wind field under the street canyon interacts with the gas-jet obstacle that forms from the surface source of heated air masses of automobile emissions. Recirculating flow forms beyond the zone of heat and mass release, i.e. street canyon. We can note that the distribution of velocity and concentration are deformed in the domain by the action of wind, which interacts with the buildings. Similarly, the others fields of component concentrations of pollutants are deformed. It allows investigating dynamics of environmental pollution under influence of various conditions: meteorology conditions and parameters of traffic flow (the number of cars of different types, traffic density and etc.). By increasing the wind velocity, the results show that the concentration of $\mathrm{CO}$ is drop around the street canyon more intensive.

\section{Conclusions}

The obtained results are agreed with the laws of physics and experimental data obtained near highway Moscow-Petersburg (Russia). Mathematical model and the results of the calculation give an opportunity to evaluate critical levels of environmental pollution and the damage from motorcar.

\section{References}

1. Sedov, L.G.: Mechanics of Continuous Medium. Science. Moscow (1976) (in Russian)

2. Maddukuri, C.S.: A Numerical Model of Diffusion of Carbon Monoxide Near Highways //Journal of Air Pollution Control Association, Vol. 32, 8 (1982) 834-836

3. Perminov, V.A.: Mathematical Modeling of Environmental Pollution by the Action of Motor Transport // Advances in Scientific Computing and Application, Science Press, Being/New York, (2004) 341-346

4. Grishin, A.M.: Mathematical Modeling Forest Fire and New Methods Fighting Them, F.Albini (ed.), Publishing House of Tomsk University, Tomsk (Russia), (1997)

5. Patankar, S.: Numerical Heat Transfer and Fluid Flow, Hemisphere Publ. Co., New York (1980) 